\title{
High Risk Bladder Cancer: Current Management and Survival
}

\author{
Anna M. Leliveld, Esther Bastiaannet, Benjamin H.J. Doornweerd, Michael Schaapveld, Igle \\ J. de Jong
}

Department of Urology (AML, BHJD, IJJ), University Medical Center Groningen, Groningen, The Netherlands, Department of Epidemiology (EB), Leiden University Medical Center, and Comprehensive Cancer Center North-Netherlands (MS), The Netherlands

\begin{abstract}
Purpose: To evaluate the pattern of care in patients with high risk non muscle invasive bladder cancer (NMIBC) in the Comprehensive Cancer Center North-Netherlands (CCCN) and to assess factors associated with the choice of treatment, recurrence and progression free survival rates.

Materials and Methods: Retrospective analysis of 412 patients with newly diagnosed high risk NMIBC. Clinical, demographic and follow-up data were obtained from the CCCN Cancer Registry and a detailed medical record review. Uni and multivariate analysis was performed to identify factors related to choice of treatment and 5 year recurrence and progression free survival.

Results: 74/412 (18\%) patients with high risk NMIBC underwent a transurethral resection (TUR) as single treatment. Adjuvant treatment after TUR was performed in $90.7 \%$ of the patients treated in teaching hospitals versus $71.8 \%$ in non-teaching hospitals $(\mathrm{p}<0.001)$. In multivariate analysis, age $(60-79$ years OR 0.40 and $>$ 80 years OR $0.1 \mathrm{p}=0.001)$ and treatment in non-teaching hospitals (OR $0.25 ; \mathrm{p}<0.001)$ were associated with less adjuvant treatment after TUR. Tumor recurrence occurred in 191/392 (49\%) and progression in $84 / 392$ (21.4\%) patients. The mean 5-years progression free survival was $71.6 \%$ (95\% CI 65.5-76.8).

Conclusion: In this pattern of care study in high risk NMIBC, $18 \%$ of the patients were treated with TUR as single treatment. Age and treatment in non-teaching hospitals were associated with less adjuvant treatment after TUR. None of the variables sex, age, comorbidity, hospital type, stage and year of treatment was associated with 5 year recurrence or progression rates.
\end{abstract}

Key words: urinary bladder neoplasms; treatment; outcomes

Int Braz J Urol. 2011; 37: 203-12

\section{INTRODUCTION}

Bladder cancer is the second most common urological cancer. Non muscle invasive forms include carcinoma in situ (CIS) and Ta/T1 carcinomas and accounts for more than half of the incidence. These tumors frequently recur, particularly in multi focal disease. Progression to muscle invasive disease is related to risk groups. The high risk group consists of patients with a high grade tumor (Grade 3) with/without concurrent CIS or CIS alone. Progression rates in this group of patients show a wide range (12 - 52\%) (1-3).

Adjuvant intravesical chemotherapy, either single immediate or serial additional instillations, is effective in decreasing local recurrence in low grade 
tumors $(4,5)$. However, these instillations do not prevent progression. In high grade tumors Bacillus Calmette Guerin (BCG) instillations are superior to intravesical chemotherapy to prevent or delay progression. For optimal efficacy BCG maintenance therapy is necessary $(1,3,6)$. If conservative treatment fails patients present with a recurrence of high risk bladder cancer with or without progression, the most common therapy is radical cystectomy. The exact timing of this cystectomy is still unclear and the subject of many studies.

Despite the presence of guidelines towards management of bladder cancer of the American and European Urological Associations, the actual management shows quite large variation. A great majority of surveyed urologists in the USA in 2003 were still reluctant to perform a cystectomy in patients with a high grade tumor who failed intravesical immunotherapy although this was described in the guidelines and a cystectomy in this group of patients gives a tumorspecific survival of $80-90 \%$ (7).

In the Netherlands a national guideline for diagnosis and treatment of bladder cancer has been available since 2009. Until that moment regional consensus based guidelines are available within the regional comprehensive cancer centers. The current clinical practice roughly matches the European Association of Urology guidelines $(8,9)$.

Pattern of care studies are used to identify disparities in patient treatment. These studies can also evaluate which tumor related, patient related or other factors determine the treatment decisions and how this could influence the course and development of the treatment outcome $(7,10,11)$.

The aim of the study was to evaluate the actual pattern of care in patients with newly diagnosed high risk NMIBC and to assess factors associated with the choice of treatment, 5 year recurrence and progression free survival rates.

\section{MATERIAL AND METHODS}

\section{Selection}

From the cancer registry of the $\mathrm{CCCN}$ a total of 535 patients were identified with a first manifestation of NMIBC in the period 1997-2002. We selected all cases with a non muscle invasive urothelial cell carcinoma stage $\mathrm{Ta} / 1 \mathrm{G} 3$ with/without CIS or isolated CIS of the bladder. Patients with metastatic disease $(n=5)$ and TxG3 $(n=8)$ were excluded. From 110 patients the medical records were not available for data collection on follow-up and recurrence. The data of these 110 patients in the $\mathrm{CCCN}$ registry showed identical characteristics and similar distribution regarding stage and grade of the tumor compared to the studied cohort. The final analytic cohort included 412 patients with high risk NMIBC of the bladder.

\section{Data Collection by the Cancer Registries}

Data were collected by the regional cancer registry of the CCCN. Within the CCCN a total of 15 hospitals participated including one University Medical Center and 3 major teaching hospitals. The nationwide Dutch network and registry of histoand cytopathology regularly submits reports of all diagnosed malignancies to the cancer registries. Trained registry personnel collect data on demography, diagnosis, staging, co-morbidity, treatment and follow-up from the medical records, including pathology and surgery reports. Treatment was coded in sequence of administration to a maximum of four treatment modalities. Vital status was established either directly from the patient's medical record or through linkage of cancer registry data with the municipal population registries. Stage was based on pathological information of primary tumor or first recurrence. CIS is only registered as CIS in cases of isolated CIS; concomitant CIS is included in the group Ta/T1G3 tumor. BCG-failure was defined as recurrent disease in patients who were treated with a first course of 6 instillations of BCG. Teaching hospitals were defined as hospitals which provide training for residents to become urologist. We analyzed the retrospective data anonymously and therefore no formal Ethics Committee approval was needed. The local oncology committees have granted permission to examine the medical files of the included patients for additional data which were collected. 


\section{Statistics}

To assess the factors associated with choice of treatment we used a logistic regression analysis. Age was studied as a continuous factor and grouped using decades. Time to local recurrence is defined as the time from diagnosis to the registered histological proven recurrence in the bladder after prior treatment. Progression time was defined as time from diagnosis to any registered recurrent tumor with progression in T-stage, arising concomitant CIS or progression to lymph node or distant metastases. Differences in 5 year Recurrence-Free Survival (RFS) and Progression-Free Survival (PFS) were assessed using the log-rank test. The effect of several factors on 5 year RFS and PFS were studied using a multivariable Cox regression analysis. As cause of death was unknown in $19.8 \%$ of the patients we choose to use relative survival. The relative survival has been shown to be a good estimator of the disease specific survival in absence of information on the cause of death or in case information on the cause of death is inaccurate (12). The relative survival estimates the net bladder cancer survival, survival in the hypothetical situation that bladder cancer is the only possible cause of death. Maximum follow-up was curtailed at 8 years. Relative excess risks of death were estimated using a multivariate generalized linear model with a Poisson error structure, based on collapsed relative survival data, using exact survival times (13).

\section{RESULTS}

\section{Treatment}

Overall, $18 \%$ of the 412 patients with a high risk NMIBC underwent a TUR as single treatment (Table-1). Seventy-seven percent of the patients received adjuvant treatment with a single immediate Mitomycin (MMC)-instillation, MMC-instillations or BCG-instillations with or without a prior single MMC-instillation (3.3\%, 39\% and 34\%, respectively). For the assessment of associating factors in the choice for TUR with adjuvant therapy 20 patients were excluded because of any other treatment (radiotherapy, cystectomy).
Table 1 - Patient characteristics, hospital type, tumor and treatment.

\begin{tabular}{|c|c|c|}
\hline & $\mathbf{N}$ & $\%$ \\
\hline \multicolumn{3}{|l|}{ Sex } \\
\hline Male & 354 & 85.9 \\
\hline Female & 58 & 14.1 \\
\hline \multicolumn{3}{|l|}{ Age } \\
\hline$<60$ & 68 & 16.5 \\
\hline $60-69$ & 121 & 29.4 \\
\hline $70-79$ & 155 & 37.6 \\
\hline$>80$ & 68 & 16.5 \\
\hline \multicolumn{3}{|l|}{ Year } \\
\hline 1997-1999 & 197 & 47.8 \\
\hline $2000-2002$ & 215 & 52.2 \\
\hline \multicolumn{3}{|l|}{ Comorbidity } \\
\hline None & 115 & 27.9 \\
\hline One & 159 & 38.6 \\
\hline Two or more & 138 & 33.5 \\
\hline \multicolumn{3}{|l|}{ Hospital } \\
\hline Non-teaching & 220 & 53.4 \\
\hline Teaching & 192 & 46.6 \\
\hline \multicolumn{3}{|l|}{ Stage } \\
\hline TaG3 & 125 & 30.3 \\
\hline $\mathrm{T} 1 \mathrm{G} 3 / 4$ & 258 & 62.6 \\
\hline CIS & 29 & 7.7 \\
\hline \multicolumn{3}{|l|}{ Treatment } \\
\hline TUR & 76 & 18.4 \\
\hline TUR with single instillation & 14 & 3.4 \\
\hline TUR with chemotherapy & 160 & 38.8 \\
\hline TUR and BCG & 142 & 34.5 \\
\hline Cystectomy & 16 & 3.9 \\
\hline Radiotherapy & 4 & 1.0 \\
\hline
\end{tabular}

$B C G=$ bacillus Calmette-Guérin $; T U R=$ transurethral resection; $C I S=$ carcinoma in situ .

As shown in Table-2, age was related to receiving adjuvant therapy (OR 0.94-95\% CI 0.91-0.97) in univariate analysis. Patients younger than 60 years received adjuvant therapy in $92.2 \%$ of the cases and only $71.3 \%$ of the elderly patients $(\geq 70$ years) did receive adjuvant therapy. Patients treated in teaching hospitals received adjuvant treatment after the TUR 
Table 2 - Transurethral resection.

\begin{tabular}{|c|c|c|c|c|c|c|c|}
\hline \multicolumn{2}{|c|}{ Variable } & \multirow[t]{2}{*}{$\mathbf{N}$} & \multirow{2}{*}{$\frac{\text { TUR plus Adjuvant }}{\%}$} & \multirow{2}{*}{$\frac{\text { Univariate Analysis }}{\mathrm{p} \text { Value }}$} & \multicolumn{3}{|c|}{ Multivariate Analysis } \\
\hline & & & & & OR & $95 \% \mathrm{CI}$ & $\mathrm{p}$ Value \\
\hline \multirow[t]{2}{*}{ Sex } & Male & 339 & 80.2 & & & & \\
\hline & Female & 53 & 83.0 & 0.6 & & & \\
\hline \multirow[t]{5}{*}{ Age } & Continuous & 392 & 80,6 & $<0.001$ & & & \\
\hline & $<60$ & 64 & 92.2 & & Ref & & \\
\hline & $60-69$ & 117 & 83.8 & & 0.4 & $0.1-1.2$ & \\
\hline & $70-79$ & 145 & 82.1 & & 0.4 & $0.1-1.1$ & \\
\hline & $>80$ & 66 & 60.6 & $<0.001$ & 0.1 & $0.04-0.4$ & $<0.001$ \\
\hline \multirow[t]{3}{*}{ Co-morbidity } & None & 108 & 81.5 & & & & \\
\hline & One & 152 & 80.9 & & & & \\
\hline & Two or more & 132 & 79.6 & 0.9 & & & \\
\hline \multirow[t]{2}{*}{ Hospital } & Teaching & 183 & 90.7 & & Ref & & \\
\hline & Non-teaching & 209 & 71.8 & $<0.001$ & 0.25 & $0.13-0.45$ & $<0.001$ \\
\hline \multirow[t]{3}{*}{ Stage } & $\mathrm{TaG} 3$ & 122 & 82.8 & & & & \\
\hline & $\mathrm{T} 1 \mathrm{G} 3 / 4$ & 241 & 78.4 & & & & \\
\hline & CIS & 29 & 89.7 & 0.3 & & & \\
\hline \multirow[t]{2}{*}{ Year } & 1997-1999 & 186 & 78.0 & & & & \\
\hline & $2000-2002$ & 171 & 83.0 & 0.206 & & & \\
\hline
\end{tabular}

$B C G=$ Bacillus Calmette-Guérin $;$ TUR $=$ transurethral resection $; C I S=$ carcinoma in situ.

in $90.7 \%$ compared to $71.8 \%$ in patients treated in non-teaching hospitals $(\mathrm{p}<0.001)$. In multivariate analysis, age (60-79 years OR 0.40 and $>80$ years OR $0.1 \mathrm{p}=0.001)$ and treatment in non-teaching hospitals (OR $0.25 ; \mathrm{p}<0.001)$ were associated with less adjuvant treatment after TUR.

\section{Progression-Free Survival}

Local recurrence was observed in 191 of the 392 patients $(48.2 \%)$ with their bladder in situ during a mean follow up of 2,7 years (range $0.1-9.2$ ). Of the 76 patients treated with TUR alone 44 (58\%) showed recurrent disease versus 147 (49\%) of the 316 patients treated with adjuvant chemo or BCG therapy showed recurrent disease (hazard ratio (HR)
0.66 (95\% CI 0.47-0.93); $\mathrm{p}=0.02$ ). Median time to local recurrence was 2.6 years (range $0.10-9.20$ ). None of the other variables sex, age, co morbidity, teaching/non-teaching hospitals, stage, year of treatment was associated with local recurrence (data not shown).

Tumor progression occurred in 84 of the 392 patients $(21.4 \%)$. The mean 5 -years progression free survival was $71.6 \%$ (95\% CI $65.5-76.8)$. In multivariate analysis disease progression showed a trend towards significance with stage (adjusted for age and type of growth) with a HR of $0.97(95 \%$ CI $0.60-1.56$ ) for T1G3/4 (as opposed to TaG3) and a HR of 0.13 (95\% CI 0.02-0.97) for CIS with a p-value of 0.1 . None of the other registered factors was statistically significant associated with progression-free survival. 


\section{Relative Survival}

During a median follow-up period of 4.5 years (range $0.1-8.0), 164$ of the 412 patients (39.8\%) died. The 5-years relative survival was $81.9 \%(95 \%$ CI 75.2-88.0). Patients with one or more co-morbidities appeared to have a lower survival (5-years relative survival $70.8 \%$ ) than patients with no co morbidity $(95.2 \% ; p=0.084)$. None of the other registered factors was significantly associated with relative survival in either univariate or multivariate analysis.

\section{BCG Failure}

Of the 142 patients initially treated with $\mathrm{BCG}$ 76 patients $(54 \%)$ failed after first course of BCG. Patients with CIS alone (42.9\%) treated with BCG failed somewhat less often than patients with $\mathrm{TaG} 3$ $(52.9 \%)$ or T1G3 $(55.8 \%)$ with or without CIS ( $p=$ $0.4)$. BCG failure appeared to occur more often in patients with two or more co-morbidities (HR $1.38 \mathrm{p}=$ $0.2)$. Histology was not associated with BCG failure. Two thirds of the failures were still non muscle invasive tumors $(53 / 76)$. Nine of the patients were treated with a cystectomy $(17 \%)$, while 26 were treated with a second course of BCG (34\%).

\section{COMMENTS}

In this pattern of care study for high risk NMIBC in the CCCN region more than $80 \%$ of patients were treated according to international standards. Only $18 \%$ of the patients were treated with a single TUR. We found hospital type and age to be associated with the choice for less adjuvant treatment. Both age and co morbidity appeared to influence progression-free and relative survival, but no other clinical factors were associated with progression, survival or BCG failure.

In 1999 the AUA (American Urological Association) Bladder Cancer Guidelines Panel recommended intravesical chemotherapy as a treatment option after endoscopic removal of low-grade Ta bladder cancer and intravesical BCG or MMC for CIS and after endoscopic removal of T1 and high grade Ta tumors (14). Later in 2004, Sylvester et al showed a lower rate of recurrence in patients who received intravesical therapy within 24 hours postoperatively.

Worldwide the preference for TUR as single treatment for high risk NMIBC decreased throughout the last decade. In a pattern of care study by Snyder et al. $40 \%$ of the patients who were diagnosed in 1995 with a high risk bladder tumor underwent resection only (11). In our cohort, a few years later, $18 \%$ did not receive adjuvant therapy.

Remarkably in a pattern of care project of the 2003 SEER program, data suggest still an underuse of intravesical therapy in patients with high risk NMIBC in the USA. Only $42 \%$ of the 350 patients with high risk NMIBC received intravesical therapy. Stage, grade, race/ethnicity and geographic region were independently associated with intravesical therapy in this subcohort (10). Interestingly in this comparable study the use of adjuvant intravesical therapy was lower in academic hospitals (31\%) as mentioned in the Editorial Comment on this study. In our study this was the opposite with adjuvant intravesical treatment in $90.7 \%$ of the patients treated in teaching hospitals versus $71.8 \%$ in non teaching hospitals.

The proportion of patients receiving adjuvant therapy in our study is still low compared to opinions expressed by urologists in survey studies conducted in 2003-2004. Witjes et al. (2006) observed that 94.6\% of the Flemish and Dutch urologists would offer high risk patients at least an instillation course, predominantly BCG (15). In a survey in 2003 in the US $18 \%$ of the urologists stated to prefer TUR only for Ta-T1 high grade tumors and $8 \%$ and $1 \%$ for concurrent CIS and CIS only respectively (7). This difference in results between a survey study and a pattern of care study might be explained by self-selection of urologists that respond to questionnaires (around 50\%) while the actual practice of all urologists was evaluated in a pattern of care study.

We found $49 \%$ (191/392) recurrences in the patients treated with adjuvant treatment after TUR. Progression rate $21.4 \%(84 / 392)$ is also similar to the figures presented in literature $(1,3,6,16-20)$. Differences in the kind of adjuvant treatment did not appear to affect the progression rate. However, in our study it is not clear what percentage of patients treated with $\mathrm{BCG}$ in fact received long term maintenance therapy. 
Bohle et al. demonstrated in a meta-analysis of comparative studies for BCG versus MMC a significant superiority for $\mathrm{BCG}$ for the prevention of tumor progression only if BCG maintenance therapy was provided (1). In a sub-analysis Sylvester et al. also showed superiority of BCG maintenance to MMC for patients with Tis (3). A clear overview of metaanalyses of studies comparing intravesical therapies and differences was published by Hall et al. in 2007 in an update of the guideline for the management of NMIBC (Stages Ta, T1 and Tis) (14). The individual patient data meta-analysis by Malmström in 2009 showed again the superiority of BCG in the prevention of recurrences (21). Prior intravesical chemotherapy was not a confounder. Differences in outcome may be explained by heterogeneity of stage and grade of the included patients, different chemotherapeutical agents which are used and different durations of therapy.

Because of concurrent CIS in the papillary tumor groups it is very difficult to draw any conclusion about the separate effect of stage. Also there is limited inter- and intra-observer agreement in histological examination which affects the appropriateness of the staging. In a review pathology study the overall conformity in stage was only $50-60 \%$, largely due to non-agreement for the low-stage tumors. Stage T1 and CIS appear to be the most difficult entities to determine while the differentiation of these tumors is important in the decision regarding conservative or more aggressive therapy (22).

The 5 -year relative survival is $81 \%$ whereas almost $40 \%$ of the patients died during follow-up. Many patients probably died of other causes than their disease. It is obvious that multiple co-morbidities are associated with a worse relative survival. A retrospective study in 2002 on T1G3 tumor patients also did not find significant differences in survival between patients treated with BCG and transurethral resection alone (23).

Many recent studies on $\mathrm{T} 1 \mathrm{G} 3$ bladder tumor treated with BCG showed recurrences of 23 to $52 \%$ $(1,6,16,17,19,20)$. The number of patients treated in those studies did not exceed 81, but the median follow-up was longer than in our retrospective study. No difference was observed between groups of patients treated with or without the maintenance protocol after the induction therapy with BCG. Our study showed similar results with a recurrence rate of $54 \%$ and a progression rate of $16 \%$ in 142 patients who were treated with BCG.

Nowadays there is a tendency towards early cystectomy for recurrent $\mathrm{T} 1$ disease during or following intravesical BCG therapy. This may be associated with better disease specific survival. Some investigators promote immediate cystectomy for $\mathrm{T} 1 \mathrm{G} 3$ tumors (24). Differences in racial or ethnic background can also be an associating factor for tumor stage at the time of cystectomy (25). In our series $5.0 \%$ of the T1G3 tumors were treated with radical cystectomy after the diagnostic TUR. This is in concordance with the results of Joudi et al. which were $7 \%$ high risk patients who underwent radical cystectomy. Finally promising salvage therapies for patients with BCG failure, unsuitable for radical cystectomy, are awaited in the near future. Yates et al. recently published an update of the application of thermo-chemotherapy, intravesical interferon-alfa and electromotive drug administration (26).

This study has limitations due to the retrospective design. We were not able to assess socio-economic factors like educational level, income, social and martial status which are not recorded in the cancer registry and could not be obtained from the medical records. Any effects of race could not be assessed as the $\mathrm{CCNN}$ region consists of founder populations with $>95 \%$ of the inhabitants being Caucasians. Also specific determining factors in individual patients or in individual hospitals were not clarified. Finally any comparison of results between treatment groups like TUR versus TUR plus adjuvant treatment can be confounded by an indication bias.

\section{CONCLUSION}

In this pattern of care study in high risk NMIBC, only $18 \%$ of the patients were treated with a single TUR. Age and treatment in non-teaching hospitals were associated with less adjuvant treatment after TUR. None of the variables sex, age, co morbidity, teaching/non-teaching hospitals, stage and year of treatment was associated with 5 year local recurrence or with progression rates. Comorbidity was associated with a lower relative survival. 


\section{CONFLICT OF INTEREST}

None declared.

\section{REFERENCES}

1. Bohle A, Bock PR: Intravesical bacille CalmetteGuérin versus mitomycin $\mathrm{C}$ in superficial bladder cancer: formal meta-analysis of comparative studies on tumor progression. Urology. 2004; 63: 682-6; discussion 686-7.

2. Herr HW: Tumor progression and survival of patients with high grade, noninvasive papillary (TaG3) bladder tumors: 15-year outcome. J Urol. 2000; 163: 60-1; discussion 61-2.

3. Sylvester RJ, van der Meijden AP, Witjes JA, Kurth $\mathrm{K}$ : Bacillus calmette-guerin versus chemotherapy for the intravesical treatment of patients with carcinoma in situ of the bladder: a meta-analysis of the published results of randomized clinical trials. J Urol. 2005; 174 : 86-91; discussion 91-2.

4. Kaasinen E, Rintala E, Hellström P, Viitanen J, Juusela $\mathrm{H}$, Rajala $\mathrm{P}$, et al.: Factors explaining recurrence in patients undergoing chemoimmunotherapy regimens for frequently recurring superficial bladder carcinoma. Eur Urol. 2002; 42: 167-74.

5. Sylvester RJ, Oosterlinck W, van der Meijden AP: A single immediate postoperative instillation of chemotherapy decreases the risk of recurrence in patients with stage Ta T1 bladder cancer: a meta-analysis of published results of randomized clinical trials. J Urol. 2004; 171: 2186-90.

6. Sylvester RJ, van der MEIJDEN AP, Lamm DL: Intravesical bacillus Calmette-Guerin reduces the risk of progression in patients with superficial bladder cancer: a meta-analysis of the published results of randomized clinical trials. J Urol. 2002; 168: 196470.

7. Joudi FN, Smith BJ, O'Donnell MA, Konety BR: Contemporary management of superficial bladder cancer in the United States: a pattern of care analysis. Urology. 2003; 62: 1083-8.

8. Oosterlinck W, Lobel B, Jakse G, Malmström PU, Stöckle M, Sternberg C, et al.: Guidelines on bladder cancer. Eur Urol. 2002; 41: 105-12.

9. van der Meijden AP, Sylvester R, Oosterlinck W, Solsona E, Boehle A, Lobel B, et al.: EAU guidelines on the diagnosis and treatment of urothelial carcinoma in situ. Eur Urol. 2005; 48: 363-71.
10. Huang GJ, Hamilton AS, Lo M, Stein JP, Penson DF: Predictors of intravesical therapy for nonmuscle invasive bladder cancer: results from the surveillance, epidemiology and end results program 2003 patterns of care project. J Urol. 2008; 180: 520-4; discussion 524.

11. Snyder C, Harlan L, Knopf K, Potosky A, Kaplan R: Patterns of care for the treatment of bladder cancer. J Urol. 2003; 169: 1697-701.

12. Sarfati D, Blakely T, Pearce N: Measuring cancer survival in populations: relative survival vs cancer-specific survival. Int J Epidemiol. 2010; 39: 598-610.

13. Dickman PW, Sloggett A, Hills M, Hakulinen T: Regression models for relative survival. Stat Med. 2004; 23: 51-64.

14. Hall MC, Chang SS, Dalbagni G, Pruthi RS, Seigne JD, Skinner EC, et al.: Guideline for the management of nonmuscle invasive bladder cancer (stages Ta, T1, and Tis): 2007 update. J Urol. 2007; 178: 2314-30.

15. Witjes JA, Melissen DO, Kiemeney LA: Current practice in the management of superficial bladder cancer in the Netherlands and Belgian Flanders: a survey. Eur Urol. 2006; 49: 478-84.

16. Gardmark T, Jahnson S, Wahlquist R, Wijkström H, Malmström PU: Analysis of progression and survival after 10 years of a randomized prospective study comparing mitomycin-C and bacillus Calmette-Guérin in patients with high-risk bladder cancer. BJU Int. 2007; 99: 817-20.

17. Gunlusoy B, Değirmenci T, Arslan M, Nergiz N, Minareci S, Ayder AR: Recurrence and progression of T1G3 transitional cell carcinoma of the bladder treated with intravesical bacillus Calmette-Guérin. Urol Int. 2005; 75: 107-13.

18. Huncharek M, Kupelnick B: The influence of intravesical therapy on progression of superficial transitional cell carcinoma of the bladder: a metaanalytic comparison of chemotherapy versus bacilli Calmette-Guerin immunotherapy. Am J Clin Oncol. 2004; 27: 522-8.

19. Margel D, Tal R, Golan S, Kedar D, Engelstein D, Baniel J: Long-term follow-up of patients with Stage T1 high-grade transitional cell carcinoma managed by Bacille Calmette-Guérin immunotherapy. Urology. 2007; 69: 78-82.

20. Shelley MD, Wilt TJ, Court J, Coles B, Kynaston H, Mason MD: Intravesical bacillus Calmette-Guérin is superior to mitomycin $\mathrm{C}$ in reducing tumour recurrence in high-risk superficial bladder cancer: a meta-analysis of randomized trials. BJU Int. 2004; 93: 485-90.

21. Malmström PU, Sylvester RJ, Crawford DE, Friedrich M, Krege S, Rintala E, et al.: An individual patient data meta-analysis of the long-term outcome of randomised 
studies comparing intravesical mitomycin $\mathrm{C}$ versus bacillus Calmette-Guérin for non-muscle-invasive bladder cancer. Eur Urol. 2009; 56: 247-56.

22. Witjes JA, Moonen PM, van der Heijden AG: Review pathology in a diagnostic bladder cancer trial: effect of patient risk category. Urology. 2006; 67: 751-5.

23. Shahin O, Thalmann GN, Rentsch C, Mazzucchelli L, Studer UE: A retrospective analysis of 153 patients treated with or without intravesical bacillus CalmetteGuerin for primary stage $\mathrm{T} 1$ grade 3 bladder cancer: recurrence, progression and survival. J Urol. 2003; 169: 96-100; discussion 100.

24. Skinner EC: The best treatment for high-grade T1 bladder cancer is cystectomy. Urol Oncol. 2007; 25: 523-5.
25. Manoharan M, Ayyathurai R, de Los Santos R, Nieder AM, Soloway MS: Presentation and outcome following radical cystectomy in Hispanics with bladder cancer. Int Braz J Urol. 2008; 34: 691-8; discussion 698.

26. Yates DR, Rouprêt M: Failure of bacille CalmetteGuérin in patients with high risk non-muscle-invasive bladder cancer unsuitable for radical cystectomy: an update of available treatment options. BJU Int. 2010; 106: $162-7$.

\section{Correspondence address:}

Dr. A.M. Leliveld

Department of Urology

University Medical Center Groningen

Hanzeplein 1, P.O. box 30.001

Groningen, 9700 RB, The Netherlands

Fax: + 31503 619-607

E-mail: a.m.leliveld@uro.umcg.nl

\section{EDITORIAL COMMENT}

The authors examined 412 patients (diagnosed between 1997-2002) within the North-Netherlands Cancer Registry for variation in the use of adjuvant therapy in patients with high risk superficial bladder cancer (defined as high grade $\mathrm{Ta} / \mathrm{T} 1$ or CIS). $82 \%$ of patients received some form of adjuvant intravesical therapy. Of patients receiving adjuvant therapy, $4 \%$ received only a single peri-operative installation, while induction BCG or chemotherapy was given in $45 \%$ and $51 \%$ of patients, respectively. It is unclear from the presented data what proportion of patients receiving induction therapy also received a peri-operative dose. Overall, these results suggest a much higher rate of compliance with published treatment guidelines than that found in similar studies in the Unites States (ref. 10 and 11 in article). Surprisingly, increasing age, but not co-morbidity, was significantly associated with lower odds of intravesical therapy use. Contrary to the findings of Huang et al. (ref. 10), the use of adjuvant intravesical therapy was greater in teaching versus non-teaching hospitals in the Netherlands.

Because the first AUA guidelines were published in 1999 (1), and the first EAU guidelines in 
2002 (ref. 8), it will be interesting going forward to re-examine these findings as further dissemination has hopefully occurred. In addition, newer guidelines by the AUA (ref. 14) and EAU (2) emphasize re-resection of high grade T1 lesions (even with muscle in the initial specimen) as well as the use of maintenance therapy. It will also be important to examine compliance with these newer guidelines. Finally, while the identification of factors associated with decreased intravesical therapy use -- age, ethnicity, or type of practice -- gives clues as to the barriers preventing implementation, the main underlying issues may be failure to disseminate existing literature, patient burden (perceived or real), and logistical issues which affect provider work-flow.

\section{REFERENCES}

1. Smith JA Jr, Labasky RF, Cockett AT, Fracchia JA, Montie JE, Rowland RG: Bladder cancer clinical guidelines panel summary report on the management of nonmuscle invasive bladder cancer (stages Ta, $\mathrm{T} 1$ and TIS). The American Urological Association. J Urol. 1999; 162: 1697-701.

2. Babjuk M, Oosterlinck W, Sylvester R, Kaasinen E, Böhle A, Palou-Redorta J, et al.: EAU guidelines on non-muscle-invasive urothelial carcinoma of the bladder. Eur Urol. 2008; 54: 303-14.

Dr. Jared Whitson

Urologic Oncology Fellow Department of Urology

University of California San Francisco E-mail:jwhitson@urology.ucsf.edu

\section{EDITORIAL COMMENT}

From a distance, it may look as if there is nothing new under the sun regarding management of non-muscle invasive transitional cell bladder cancer. We use urine cytology, transurethral resection, intravesical doxorubicin or mitomycin and BCG for more than 35 years and they remain our main weapons for fighting not only costly (due to innumerous recurrences), but unpredictive and if progressive often fatal disease.

At a closer look, the field of this disease has changed quite significantly in recent years. Without considering new markers, drugs and techniques which are already available it may look promising in the future (studies evaluating screening with combinations of new markers like FISH analysis of exfoliated cells in urine, proteomics, fluorescence blue light cystoscopy and resection, gentamycin, microwave heated or electromotive intravesical drug application). Main change has been in standardization and systematization of our approach to patients with this disease. Those changes took place in recent years and guidelines (AUA, NCCN and EAU) are all freely available from their respective web-sites. AUA guidelines are quite narrative and give reasoning behind suggestions, NCCN produces and every few months updates short and up to a point graphic algorithms - decision trees and EAU may has produced (in their last, 2009 update) most precise and straightforward set of rules for dealing with nonmuscle invasive bladder cancer.

EAU rules seem to have almost taken "art" out of medicine in this area and "science" has almost completely taken over. Is this good? The present article by Leliveld and coworkers shows us the importance of this aspect. Although titled "High risk bladder cancer: current management and survival", they report only $35 \%$ of patients who were only treated some 8 years ago (in 2002), in a western European country, according to present regional (EAU) guidelines. This means $65 \%$ of high risk superficial bladder cancers have not received immunotherapy (BCG), which they, according to present 
guidelines, should have received. Although the authors in discussion address this issue and cite one, 8 year old study, which claims no survival advantage for immunotherapy group and this may be debatable, the fact still remains solid: majority of patients were at the time of study not treated according to present guidelines. Some patients may be unsuitable (too old etc.) for treatment according to guidelines and guidelines have room for improvement with specifically addressing those marginal groups. Perhaps some patients may be in a registered clinical trial (this is not reported). But otherwise - current management of high risk bladder cancer should not be as was mentioned in this study. If not for other reasons, guidelines take a lot of hesitation out of our mind and help us a lot in ultimate question with high risk patients - i.e. to proceed with cystectomy or continue conservative treatment? Decision for cystectomy is a difficult one and it is universally accepted it is often made too late (1). Relatively rigid guidelines offer a highway towards this decision, if and when needed. Although guidelines can and will change with time, obeying them is a first step towards improving outcomes, which for bladder cancer seem to remain the same for decades with no improvement. Only years after we have standardized our approach and try to follow guidelines with each individual patient, SEER and other cancer statistics may and will reflect improvements.

\section{REFERENCES}

1. Soloway MS, Hepps D, Katkoori D, Ayyathurai R, Manoharan M: Radical cystectomy for BCG failure: has the timing improved in recent years? BJU Int. 2010; 10. doi: 10.1111/j.1464-410X.2010.09830.x.

Dr. Tine Hajdinjak

Division of Urology, Department of Surgery Murska Sobota General Hospital Murska Sobota, Slovenia E-mail: tine.hajdinjak@gmail.com 